1. THE OBSERVATIONS 


\title{
INTERSTELLAR MOLECULES - THE OPTICAL REGION
}

\author{
D. McNALLY \\ University of London Observatory, Mill Hill Park, London N.W.7, England
}

Considerable attention has recently been given to the observation of interstellar molecules by radio methods. Interstellar molecules have also been given a boost because of excitation by the $3 \mathrm{~K}$ black body radiation field (to use a convenient description). Nevertheless optical studies of interstellar lines have reached a very low ebb and, with brighter moments, have remained so since the work of Adams (1949).

The years 1937-1942 were the exciting period for optical studies of interstellar molecular lines. It is interesting to note that it was not molecular lines as such that led to the suspicion that molecules might exist in interstellar space but it was the discovery of the diffuse features at 5780, 5797, 6284 and 6614 by Merrill (1934) that led Russell (1935) to speculate on the possibility of their existence. It was Swings and Rosenfeld (1937) who stressed that likely interstellar molecules would be $\mathrm{CH}, \mathrm{OH}$, $\mathrm{NH}, \mathrm{CN}$ and $\mathrm{C}_{2}$. Dunham, Adams and McKellar did much of the work of identifying interstellar lines in the period 1937-1940 and indeed McKellar (1941) had derived an excitation temperature of $2-3 \mathrm{~K}$ for $\mathrm{CN}-$ a value subsequently rediscovered.

\section{Sharp Molecular Lines}

Lines of the diatomic molecules $\mathrm{CH}, \mathrm{CH}^{+}$and $\mathrm{CN}$ have been detected by optical methods. The wavelengths of these lines lie in the spectral range 3137-4300 $\AA$ and no sharp molecular lines have been detected to the red of the $4300.30 \AA$ line of $\mathrm{CH}$. These lines are listed in Table I.

A detailed discussion of the interstellar lines appearing in the spectrum of $\zeta$ Oph (HD 149757) has been given by Herbig (1968) and Herbig's equivalent widths for the molecular lines are given in Table I as examples. It is to be noted that $\zeta$ Oph is a star exhibiting well developed interstellar lines and could be regarded as a classic example. (For comparison the equivalent widths of the $\mathrm{Ca}^{+}$lines at $3933.66 \AA$, $3968.47 \AA$ and the $\mathrm{Na}$ lines at $5889.95 \AA, 5895.92 \AA$ are $34.2,21.3 ; 239,189$ respectively for the same star.) For $\mathrm{CH}$ Herbig obtains $\mathrm{N}$ (molecules $\mathrm{cm}^{-2}$ ) $=4 \times 10^{13}$ and for $\mathrm{CN}$ he finds $\mathrm{N}=4.3 \times 10^{12}$ (for comparison, the sodium value for $\mathrm{N}$ is $4.9 \times 10^{13}$ ). Regrettably work of this quality has only been published for one star. Abundances of $\mathrm{CH}^{+}$have been held up because of the lack of an oscillator strength.

The work on $\zeta$ Oph has been further extended by Bortolot and Thaddeus (1969). By adding several spectra of $\zeta$ Oph together they have been able to detect the line of $\mathrm{C}^{13} \mathrm{H}^{+}$at $4232 \AA$. By comparing equivalent widths they found that the abundance ratio of $\mathrm{C}^{12}$ to $\mathrm{C}^{13}$ is 82 , nearly the terrestial value of 89 .

The wider aspects of molecular studies are not well catered for. It would be of very great interest to establish norms and variation of behaviour for the molecular lines. However, as shown in Table II the molecular lines are not well observed. It 


\section{TABLE I}

Interstellar molecular lines

\begin{tabular}{llcc}
\hline Wavelength $(\AA)$ & Molecule & Classification & $\begin{array}{c}W(\zeta \text { Oph) } \\
\text { in } \mathrm{m} \AA\end{array}$ \\
\hline 3137.53 & & & 4.0 \\
3143.15 & & $\mathrm{C}^{2} \Sigma^{+}-\mathrm{X}^{2} \Pi$ & 7.4 \\
3146.01 & $\mathrm{CH}$ & & 5.0 \\
3447.08 & & $\mathrm{~A}^{1} \Pi-\mathrm{X}^{1} \Sigma$ & 3.7 \\
3579.02 & & & 7.2 \\
3745.31 & $\mathrm{CH}^{+}$ & & 3.4 \\
3874.00 & & & 9.2 \\
3874.61 & & $\mathrm{~B}^{2} \Sigma^{-}-\mathrm{X}^{2} \Pi$ & - \\
3875.76 & & & - \\
3876.30 & $\mathrm{CN}$ & & 3 \\
3876.84 & & & 5.9 \\
3878.77 & & $\mathrm{~B}^{2} \Sigma^{-}-\mathrm{X}^{2} \Pi$ & 5.6 \\
3886.41 & & & 13.3 \\
3890.21 & $\mathrm{CH}$ & $\mathrm{A}^{1} \Pi-\mathrm{X}^{1} \Sigma$ & 27.4 \\
3957.70 & & $\mathrm{~A}^{2} \Delta-\mathrm{X}^{2} \Pi$ & 20.5 \\
4232.54 & $\mathrm{CH}$ & \\
4300.32 & $\mathrm{CH}$ & & \\
& & &
\end{tabular}

TABLE II

The observation of molecular lines

\begin{tabular}{llc}
\hline Wavelength $(\AA)$ & Molecule & $\begin{array}{l}\text { No. of stars } \\
\text { observed }\end{array}$ \\
\hline 3173.53 & & 1 \\
3143.15 & & 2 \\
3146.01 & $\mathrm{CH}$ & 1 \\
3447.08 & & 1 \\
3579.02 & & $-^{\mathrm{a}}$ \\
3745.31 & $\mathrm{CH}^{+}$ & 5 \\
3874.00 & & 5 \\
3874.61 & & 14 \\
3875.76 & & 2 \\
3876.30 & $\mathrm{CN}$ & 1 \\
3876.84 & & 1 \\
3878.77 & & $-\mathrm{a}$ \\
3886.41 & & 5 \\
3890.21 & $\mathrm{CH}$ & $-\mathrm{a}$ \\
3957.70 & & 33 \\
4232.54 & $\mathrm{CH}$ & 65 \\
4300.32 & $\mathrm{CH}$ & 42 \\
\hline
\end{tabular}

$-^{\mathrm{a}}$ means insufficient evidence presented to ascertain the number of stars observed 
will be seen from Table II that only the lines of $\mathrm{CH}^{+}$at $3957.70,4232.54$ and the line of $\mathrm{CH}$ at $4300.32 \AA$ have been extensively observed to an extent that could be considered adequate as an overlap between different surveys. Table II refers to published values - more material remains unpublished or unreduced.

However, one cannot make predictions about the nature of the interstellar gas from observations of single stars. For such purposes one needs data on a great number of stars. At first sight the information of Table II might be thought to give a possible starting point. However, this is not the case. If one wished to compare $\mathrm{CH}, \mathrm{CH}^{+}$and $\mathrm{CN}$ in the same group of stars the group would number eleven. Of these eleven stars there would be insufficient information to make similar comparisons in each case. Information of considerable value could be obtained if the detailed distribution on the sky of molecular species could be traced. For this purpose it is necessary to have equivalent width measurements. Measurements of intensity are rather less satisfactory. It is improbable that all data would be derived from a single source. Therefore the work of different authors will require reduction to a common scale (this is particularly the case for the diffuse features) and so requires considerable overlap. In the case of interstellar molecules the necessary data does not exist. There are about 20 equivalent widths for molecular lines in existence and 15 of these are in Table $I$. There is no possibility of intercomparison of authors much less of any possibility of rational astrophysics. The biggest single body of unified data remain Adams' eye estimates of intensity.

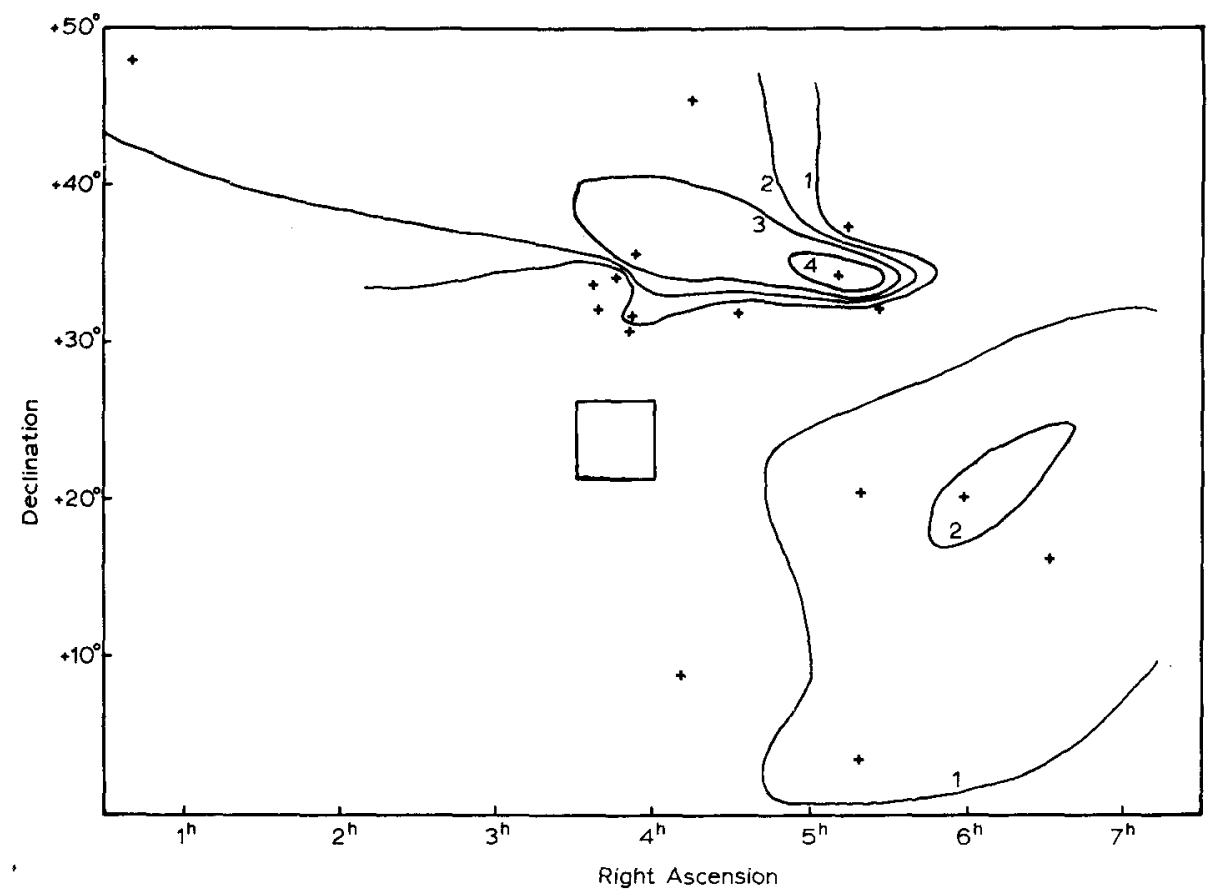

Fig. 1. Lines of equal intensity (eye estimates) for $1<\alpha<7 \mathrm{~h}, 0<\delta<50^{\circ}$ of the $\mathrm{CH}^{+}$line at $4232.54 \AA$. 


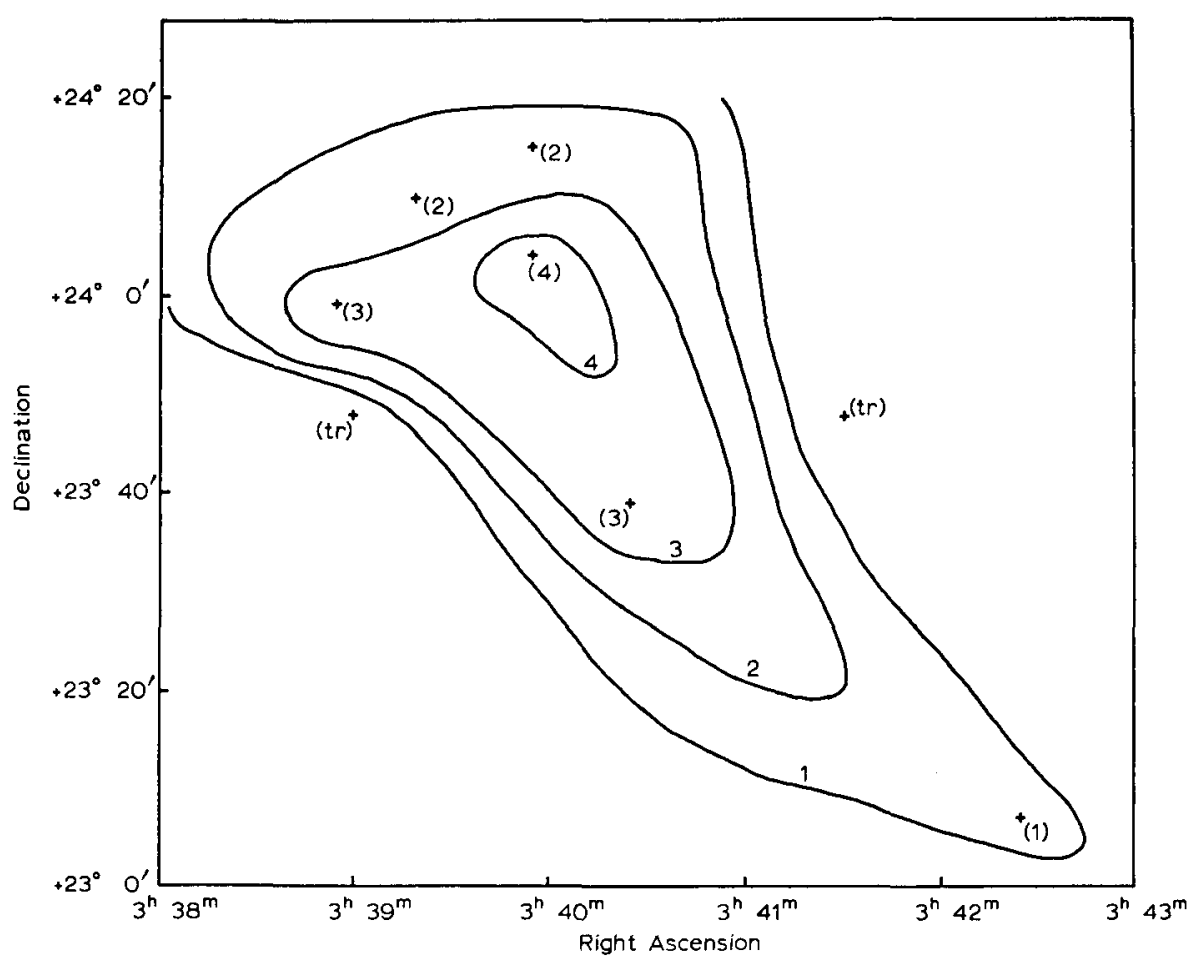

Fig. 2. Lines of equal intensity (eye estimates) for $3 \mathrm{~h} 38 \mathrm{~m}<\alpha<3 \mathrm{~h} 43 \mathrm{~m}, 23^{\circ}<\delta<24^{\circ} 20^{\prime}$ of the $\mathrm{CH}^{+}$line at $4232.54 \AA$. This region is that enclosed by the square in Figure 1 .

One can make use of Adams' material to show that it is possible to form some idea of distributions of a molecular species. The line of $\mathrm{CH}^{+}$at $4232.54 \AA$ was selected for this purpose and lines of equal eye estimate of intensity were drawn for the region $1<\alpha<7 \mathrm{hr}, 0<\delta<50^{\circ}$ as in Figure 1.

The region enclosed by the square in Figure 1 is shown enlarged in Figure 2. Of these two figures the second is the more believable. These figures demonstrate that it is possible to draw in contours but the coverage of stars on the sky is so erratic that a considerable allowance must be made for personal whimsicality in drawing the contours. I have found that before comparison can be made of contours for different species it is advisable to use the same basic group of stars since contours may be different for different groups of stars in the same area (on the basis of similar studies for the diffuse lines). Again this imposes severe restrictions on existing data.

Analyses of this type would be useful were it possible to arrive at a consistent picture since the distribution of species could then be intercompared and also compared with distributions of atoms and molecules found by other optical and radio studies. However, to obtain the necessary material an impractically large amount of telescope time would be required, though it should be feasible to obtain such material for small regions of the sky. The value of such a study cannot be overemphasised. 
TABLE III

Comparison between molecular species found under interstellar and cometary conditions

\begin{tabular}{|c|c|c|c|c|c|c|c|}
\hline Parent species & $\mathrm{H}_{2}$ & $\mathrm{CO}_{2}$ & $\mathrm{HCN}$ & $\mathrm{H}_{2} \mathrm{O}$ & $\mathrm{H}_{2} \mathrm{CO}$ & $\mathrm{NH}_{3}$ & $\mathrm{CH}_{4}$ \\
\hline Interstellar & & & $\mathrm{HCN}$ & $\mathrm{H}_{2} \mathrm{O}$ & $\mathrm{H}_{2} \mathrm{CO}$ & $\mathrm{NH}_{3}$ & \\
\hline
\end{tabular}

Cometary

\begin{tabular}{llllll} 
& & & & \multicolumn{3}{c}{$\mathrm{NH}_{2}$} \\
$\mathrm{CO}_{2}{ }^{+}$ & & & & & \\
$\mathrm{CO}^{+}$ & $\mathrm{CN}$ & $\begin{array}{l}\mathrm{OH} \\
\mathrm{OH}^{+}\end{array}$ & $\left(\mathrm{CO}^{+} ?\right)$ & $\mathrm{NH}$ & $\mathrm{CH}^{+}$
\end{tabular}

There are parallels to be diawn between interstellar and cometary spectra. There is a considerable amount of overlap in the species detected, but with certain exceptions - the most notable being the lack of detection of $\mathrm{C}_{2}$ or $\mathrm{C}_{3}$ in interstellar space. On the hypothesis (now somewhat in eclipse) that graphite was a fundamental constituent of interstellar grains it might have been reasonably expected that $\mathrm{C}_{2}$ and $\mathrm{C}_{3}$ would exist in the interstellar gas. The comparison between the interstellar and cometary cases is presented in Table III.

The parent molecular species are first given and derivatives are then listed (a) for the interstellar case and (b) for the cometary case. The similarities between the two cases could suggest that interstellar and cometary molecules are derived from the same type of material, i.e. supporting a degradation type of argument such as that presented by Herbig (1969) rather than synthesis so much favoured in the past. However, this evidence is at best circumstantial and is far from critical. An interesting observation is that in cometary spectra the $\mathrm{CN}$ lines appear first.

\section{Diffuse Molecular Lines}

The interstellar spectrum contains up to 25 diffuse lines. So far they remain unidentified though several exotic schemes have been put forward. I am claiming them for molecular species since recent studies give some grounds for hoping that identifications will be forthcoming in the not too distant future. Herbig has produced a list of these lines which appear in the spectrum of HD 183143 (another classic example for interstellar spectra), which is given in Table IV (by kind permission of Prof Herbig). It is at once clear that some of these lines are very wide indeed and that the remainder are just diffuse. Division can clearly be made into two such broad categories.

These lines are conspicuous. They are not so obvious as $\mathrm{H}$ and $\mathrm{K}$ lines of $\mathrm{Ca}^{+}$or the $D$ lines of sodium but the lines at $4428,5780,5797$ and $6284 \AA$ are more obvious than the $4226.73 \AA$ line of $\mathrm{Ca}$ and any molecular line. The origin of these lines has defied discovery since 1934. However, one can make several observations. 
TABLE IV

Wavelengths, widths and equivalent widths of the diffuse lines in HD 183143

\begin{tabular}{lcl}
\hline Wavelength $(\AA)$ & $\begin{array}{l}\text { Total width at half depth } \\
(\AA)\end{array}$ & $\begin{array}{l}\text { Equivalent width } \\
(\AA)\end{array}$ \\
\hline 4428 & 20 & 5 \\
4501.2 & 3 & 0.3 \\
4726.7 & 4 & 0.2 \\
4762.3 & $4:$ & 0.3 \\
4883 & $40:$ & $4:$ \\
5362 & 5 & 0.2 \\
5420 & $10:$ & - \\
5448 & 14 & 0.6 \\
5487.31 & 5 & 0.3 \\
5705.17 & 4 & 0.3 \\
5778 & 17 & $1.0:$ \\
5780.39 & 2.5 & 0.8 \\
5797.01 & 1.2 & 0.4 \\
5844 & 4 & 0.1 \\
5849.78 & 1 & 0.1 \\
6010.75 & 5 & 0.2 \\
6175 & 30 & 3 \\
6195.95 & $<1$ & 0.1 \\
6203.06 & 2 & 0.4 \\
6269.74 & 1.5 & 0.4 \\
6283.90 & 4 & 2 \\
6376.08 & 2 & 0.1 \\
6379.21 & 1 & 0.2 \\
6613.62 & 1 & 0.4 \\
6660.64 & 1 & 0.1 \\
& & \\
\hline & &
\end{tabular}

By kind permission of Dr G. H. Herbig.

(1) The lines seem to fall into two broad categories. Could this imply two or more species producing the lines?

(2) Either the lines such as $4428,5780,5797,6284$ are produced by fairly abundant species or the oscillator strengths for these transitions are large. I prefer the first hypothesis since large oscillator strengths usually go with sharp lines.

W. B. Somerville and I have looked at the frequency relations between diffuse features. We found that the very broad lines (such as $4428,4883,6175$ ) formed a sequence and that if the line at 4501 were omitted only two further frequencies had to be used to explain the remaining lines. However, the line at $4501 \AA$ rather upsets this scheme. We only feel justified in claiming a relationship between the very broad lines. This experience makes me wary of identifications based on numerical frequency differences.

I feel that the question of diffuse lines has been done a disservice by the weight of attention given to the line at $4428 \AA$. The line at $6284 \AA$ is equally if not more conspicuous though unfortunately coincides with minor atmospheric absorption features. 
I feel that attempts to identify the line at $4428 \AA$ in isolation may be misplaced endeavours.

In order to avoid a clash of material with Professor Herzberg's paper, I will confine my attention to a suggestion made by Johnson (1970) of a possible source of these lines. He suggests that the molecule

$$
\mathrm{C}_{46} \mathrm{H}_{30} \mathrm{Mg} \mathrm{N} \mathrm{N}_{6}
$$

by name Bis-pyridyl-magnesium-tetrabenzoporphine, may be the source of many of the interstellar diffuse features. The identification is based on certain coincidences in wavelength with lines which could be produced by this molecule. A possible structure for this molecule is illustrated in Figure 3.

Such a large molecule is startling. Having only recently become used to the excitements of molecules composed of up to four atoms, one might be prepared for anything but such a large proposed molecule still seems implausible. Johnson states that

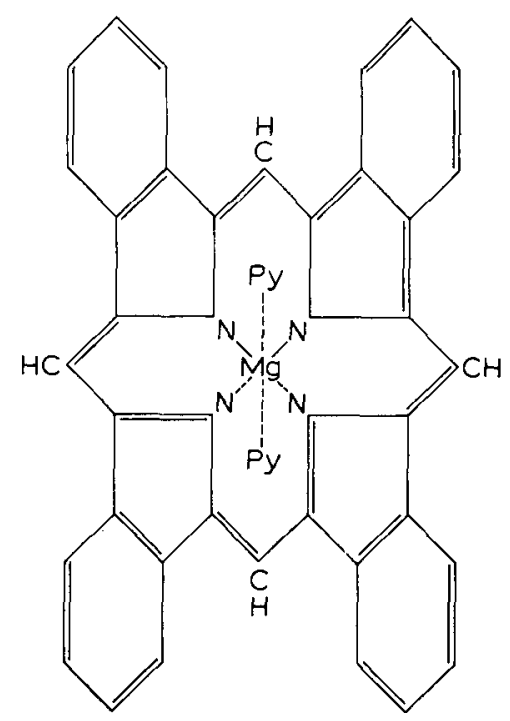

Fig. 3. A possible structure for $\mathrm{C}_{46} \mathrm{H}_{30} \mathrm{MgN}_{6}$. Py $=$ Pyridine.

such a molecule accounts for some 16 of the known diffuse features as far as wavelength is concerned. Nevertheless, such a large molecule must be fairly rare with an abundance (with respect to hydrogen) of about $10^{-11}$ or $10^{-12}$ on grounds of reasonable expectation (i.e. lying between the abundances of diatomic molecules and grains). However, assuming that such molecules accounted for all the interstellar carbon a density as high as $2 \times 10^{-5}$ could be tolerated though would be unlikely.

If Johnson is correct his spectrum would have to reproduce line profiles and also 
give reasonable excitation characteristics. For example assuming the lines 4428 and $6284 \AA$ to be produced by this molecule one would expect that the variation of equivalent width of these lines from star to star would give information on the conditions of excitation.
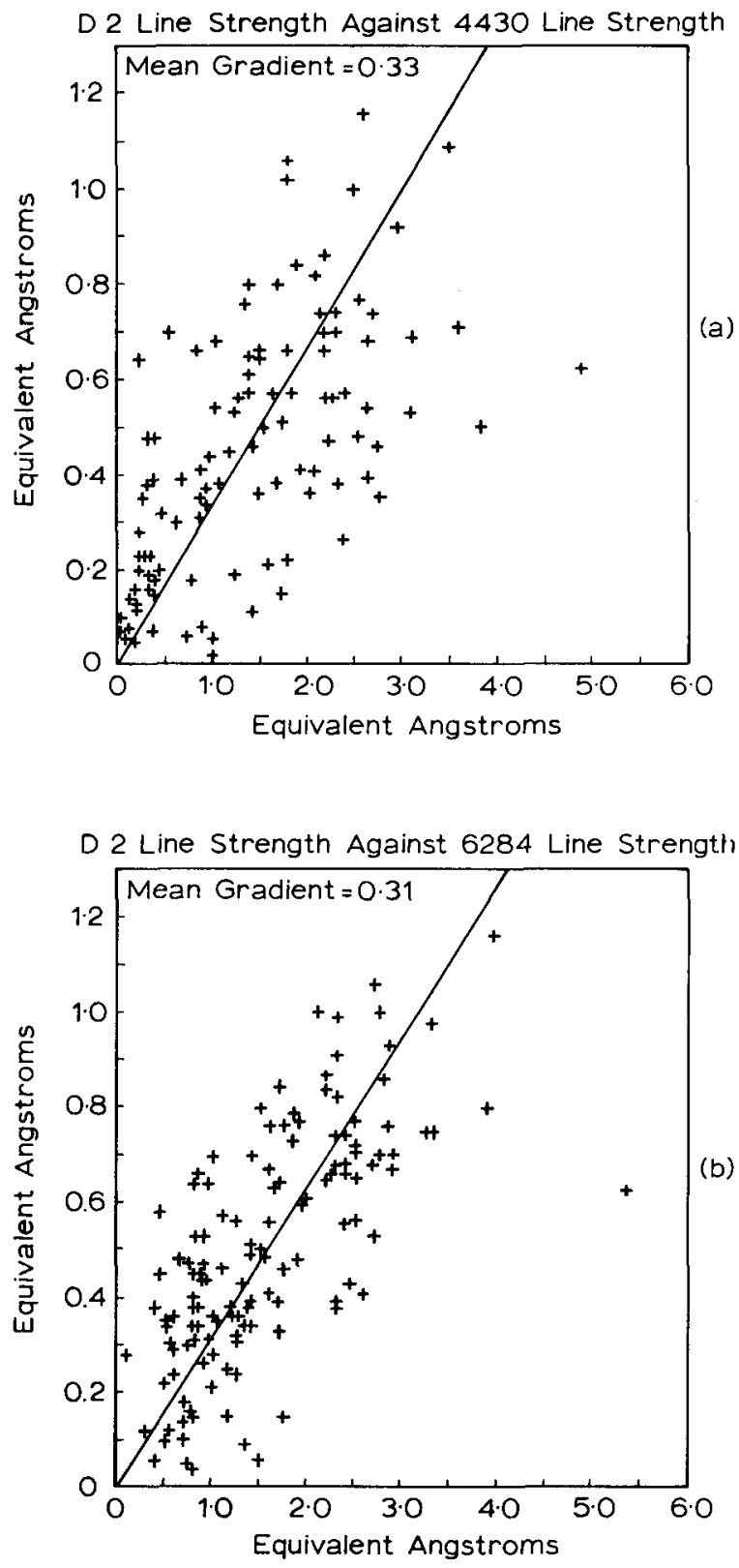

Fig. 4. 


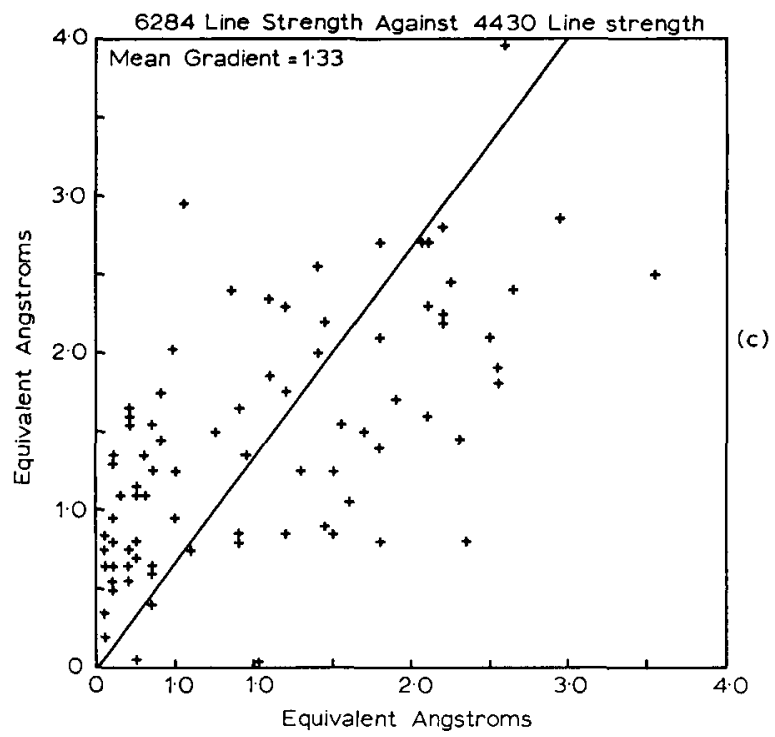

Fig. 4. (a) Correlation of equivalent widths: $D 2$ vs 4428 . (b) Correlation of equivalent widths: $D 2$ vs 6284 . (c) Correlation of equivalent widths: $6284 \mathrm{vs} 4430$. The line is through the mean point and the origin at the zero of equivalent width.

Figure $4 \mathrm{a}$ shows correlation of the line at $4428 \AA$ with the $D 2$ line of $\mathrm{Na}$, Figure $4 \mathrm{~b}$ shows the correlation of the line at $6284 \AA$ with the $D 2$ line. Both show a correlation in the expected way. Figure $4 \mathrm{c}$ shows the correlation of the 4428 and $6284 \AA$ lines. Clearly the line drawn through the mean of all equivalent widths and the origin at zero equivalent width does not adequately represent the correlation. The scatters in all three correlations are very large. The data for these correlations comes from data of different surveys which has been standardised. Standardisation is necessary since equivalent widths for the diffuse features vary markedly with the investigator. While scatter is introduced by the standardisation procedure these diagrams suggest a large variation in the conditions of relative excitation of these lines if they are produced in the same molecule, which is a curious result for optical studies.

The correlation illustrated in Figure 5 of the diffuse lines at 5780 and $5797 \AA$ shows a much reduced scatter and correlates in the expected manner. The line has a slope of 0.34 and such a slope is found with great consistency for this pair of features. The correlation of these two lines with other diffuse features shows a return to large scatters.

Figure 6 showing the equivalent width plot of the line at $6614 \AA$ against the $D 2$ line of sodium indicates almost no correlation - this is the consistent verdict of any correlation attempt with the line at $6614 \AA$ though lack of data is probably the largest contributory effect in this case.

The correlation studies cited here do not bring much support to Johnson's interesting proposal. They rather suggest that the diffuse features may result from at least 
three different species. However, preliminary contour studies suggest a similar distribution on the sky for the lines at 4428 and $6284 \AA$ and so similar spatial distributions of carrier or carriers.

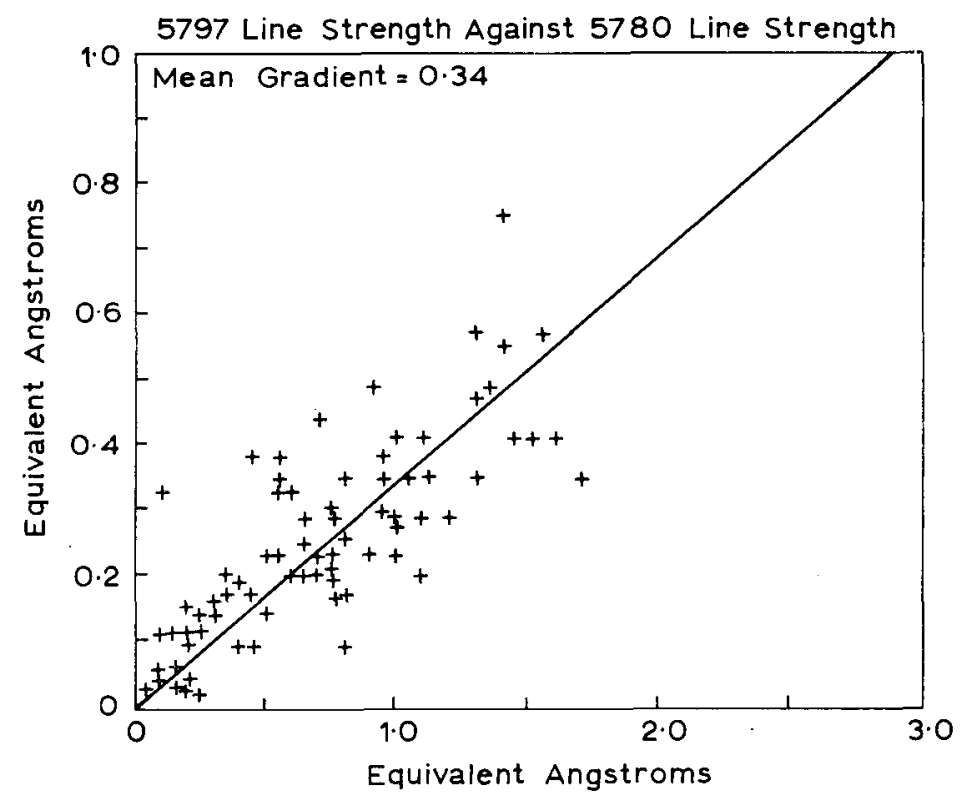

Fig. 5. Correlation of equivalent widths: 5797 vs $\mathbf{5 7 8 0}$. The line is drawn as in Figure 3.

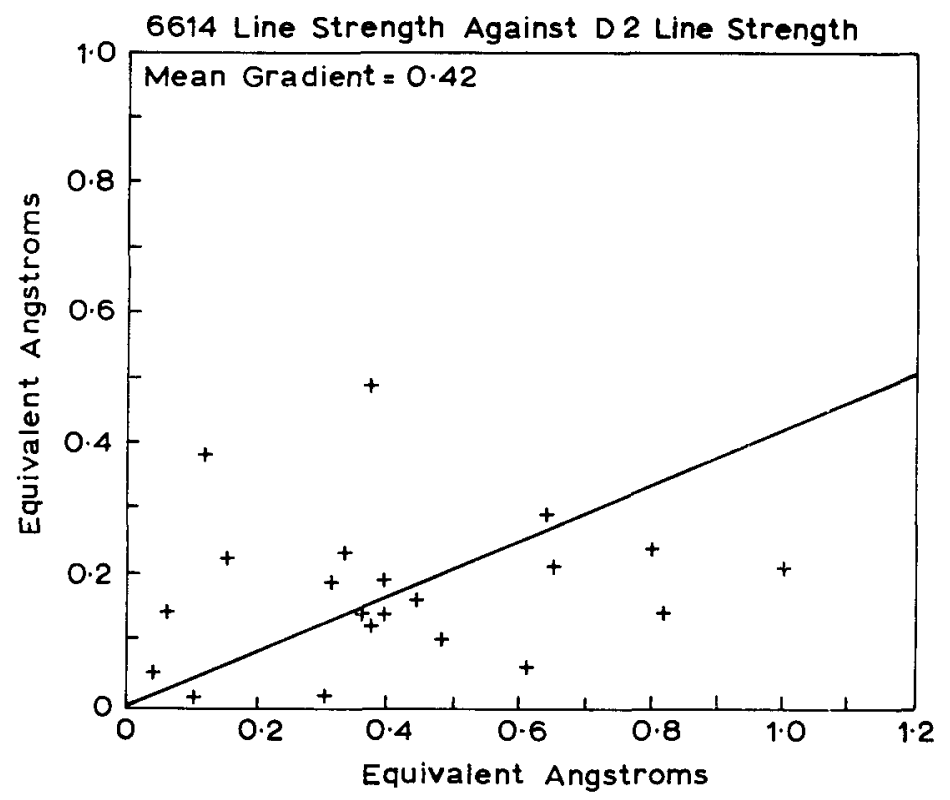

Fig. 6. Correlation of equivalent widths: 6614 vs $D 2$. The line is drawn as in Figure 3. 


\section{Conclusion}

The situation regarding interstellar molecules is poor. Only rarely are equivalent widths obtained and then usually in penny numbers. Extensive survey work over the whole sky is probably not feasible (from the point of view of availability of telescope time) but certain small areas of sky would be worth detailed investigation. The optical studies could then be used to get some idea of the distribution of species over the sky and so help in many problems in the structure of the interstellar medium. Detailed star by star studies could provide useful calibrations. Different surveys should have sets of stars in common to help standardise equivalent width scales. The observations of molecular lines in the optical region of the spectrum provides no base for comparison with either the radio or infra-red work now being done.

\section{References}

Adams, W. S.: 1949, Astrophys. J. 109, 354.

Bortolot, V. J., Jr. and Thaddeus, P.: 1969, Astrophys. J. 155, L17.

Herbig, G. H.: 1968, Z. Astrophys. 68, 243.

Herbig, G. H.: 1970, Mem. Soc. Roy. Sci. Liege, 5th Series 19, 13.

Johnson, F.: 1970, Abstracts of 132nd Meeting of AAS, abstract 6.06.

McKellar, A.: 1941, Publ. Dominion Astrophys. Obs. Victoria 7, 251.

Merrill, P. W.: 1934, Publ. Astron. Soc. Pacific 52, 187.

Russell, H. N.: 1935, Monthly Notices Roy. Astron. Soc. 95, 610.

Swings, P. and Rosenfeld, L.: 1937, Astrophys. J. 95, 270.

\section{DISCUSSION}

Sagan: The reference to $\mathrm{F}$. Johnson's porphyrin derivative, $\chi$, deserves some comment. I think there are excellent reasons to propose that complex organic molecules are produced in the interstellar medium. I'm prepared to believe that molecules with high stability to ultra-violet radiation and low energy cosmic rays, such as the porphyrins, might be preferentially present even if produced very slowly - by a kind of molecular natural selection. And I'm even prepared to accept the easily derived implication of Johnson's work that $\sim 1 \%$ of interstellar $\mathrm{C}$ atoms are tied up in big molecules. But I'm not prepared to accept the identification of $\chi$ for the following reason. The proposed molecule is said to explain the 15 strongest unidentified diffuse interstellar lines to $t 2 \AA$ in central frequency and some comparable accuracy in equivalent width. It is a $\mathrm{Mg}$-chelated tetra-pyrrole with benzenes on each pyrrole group, and pyridines attached to the $\mathrm{Mg}$ ion. Now what happens to the absorption spectrum if I make a small change - a $\mathrm{CH}_{3}$ for an $\mathrm{H}$ on a benzene, say, or $\mathrm{Fe}$ for $\mathrm{Mg}$ ? All the lines vary, we know from chlorophyll chemistry, often by much more than $\pm 2 \AA$, but retaining a comparable line strength. Where are the lines of such molecules? It seems very unlikely that no molecules closely related to $\chi$ are formed, unless we were willing to accept the hypothesis of biological origin for interstellar organic molecules. This hypothesis does not appear to be compelling at the present time.

Brand: At Edinburgh, image tube observations with the $36^{\prime \prime}$ spectrograph have confirmed our first findings that there exists a blue emission wing on the $\lambda 4430$ feature, of height $1-2 \%$.

In addition, recent measurements using a Wollaston prism indicate that there is differential polarisation across the $\lambda 4430$ feature.

These observations should limit the possible number of models for diffuse features.

Bidelman: Dr. C. K. Kumar of the University of Michigan Observatory has recently drawn attention to the fact that the energy difference between the valence and conduction bands of the semi-conductor $\mathrm{SiC}$ corresponds to a wavelength very close to $\lambda 4430 \AA$. He has thus suggested that the energy levels associated with the several unidentified interstellar features arise in SiC crystals contaminated with various impurities. 